\section{Sexual Surrogate Therapy}

Bruce Rybarczyk

Department of Psychology, Virginia

Commonwealth University, Richmond, VA, USA

\section{Synonyms}

Surrogate partner therapy

\section{Definition}

A method or treatment, seldom employed in the United States, which is sometimes recommended for individuals with traumatic brain injury and other neurologic disorders that significantly alter or compromise sexual functioning. Advocates view the use of surrogate therapy as an important aspect of a holistic approach to rehabilitation aimed at improving quality of life and fulfilling basic human intimacy needs. When this therapy is practiced in a professional manner a client, a trained sexual therapist, and a trained surrogate partner will form a three-person therapeutic team. The surrogate participates with the client in structured and unstructured experiences that are designed to build client self-awareness and skills in the areas of physical and emotional intimacy. These therapeutic experiences include relaxation techniques in intimate situations, effective communication, sensual and sexual touching, and social skills training. The therapist is crucial to the process in terms of helping the client learn from the experiences and generally supervising the process. When the client is higher functioning, the goal is to transition to a mutually initiated, nonsurrogate relationship. While there are several case reports in the scientific literature, there are no treatment studies addressing the efficacy of surrogacy treatment to date. A 2012 movie, The Sessions, starring Helen Hunt as the therapist, provided a sensitive portrayal of the real-life sexual surrogacy experience of Mark O'Brien, a poet paralyzed from the neck down due to polio.

\section{References and Reading}

Aloni, R., \& Katz, S. (2003). Sexual difficulties after traumatic brain injury and ways to deal with it. Springfield: Charles C. Thomas. 